\title{
Role of Classical Complement Activation in SARS-Cov-2 Infection; A Case Report of COVID-19 in A Patient with Saddle Pulmonary Embolism
}

\section{Teklie Y*, Campdesuner V, George J and Villasmil R}

Sarasota Memorial Health Care System, Sarasota, United States

*Corresponding author: Yeshanew Teklie, MD, Sarasota Memorial Health Care System, Sarasota, United States

Received date: 23 September 2020; Accepted date: 02 October 2020; Published date: 07 October 2020

Citation: Teklie Y, Campdesuner V, George J and Villasmil R (2020) Role of Classical Complement Activation in SARS-Cov-2 Infection; A Case Report of COVID-19 in A Patient with Saddle Pulmonary Embolism. J Med Case Rep Case Series 1(4): https://doi.org/10.38207/jmcrcs20201046

Copyright: (C) 2020 Teklie Y. This is an open-access article distributed under the terms of the Creative Commons Attribution License, which permits unrestricted use, distribution, and reproduction in any medium, provided the original author and source are credited.

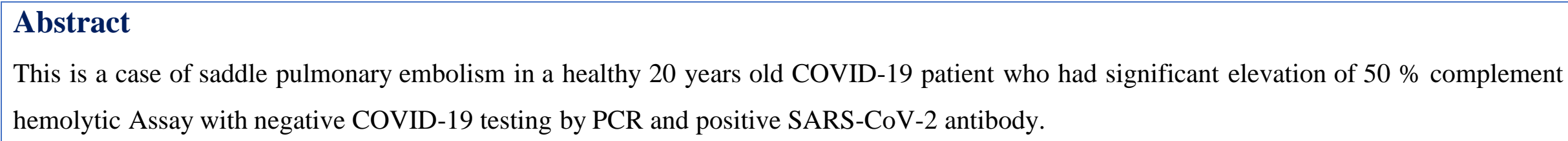

\section{Introduction}

The complement system is an agent pro-inflammatory host defense mechanism that is part of both the night and adaptive immunity. Per Richard Stienm [1], on UpToDate, the complement system plays a vital role in instructing the adaptive immune response and removal of damaged cells, tissue regeneration, and angiogenesis. However excessive complement activation can lead to cellular damage and cell lysis.

Per Wolfeam Ruf on his publication on 'the blood', complement and coagulation systems play a role in eliminating perivascular and intravascular microorganisms in the process of cold Immunothrombosis [2]. Activated complement factor 3, specifically, stimulates platelets through $\mathrm{C} 3 \mathrm{a}$ receptor signaling and thereby amplifies thrombus formation. Activation of the classicalcomplement pathway by therapeutic anti-thymocyte globulin preparations rapidly enhances monocyte tissue factor procoagulant activity, according to the article.

Based on an article by Maurizio Costabile, the classical complement activation is measured by the $50 \%$ complement hemolytic assay ( $\mathrm{CH}$ 50) which tests the functional capacity of serum complement components of the classical pathway to lyse sheep red blood cells precoated with rabbit anti-sheep red blood cell antibody [3]. During classical complement is activation by antigen-antibody complex, $\mathrm{CH}$ 50 will be high while complement component deficiencies result in low $\mathrm{CH}$ 50. Per Maurizio, individual complement components including complement 3 and complement 4 can be quantified, however, this does not provide any information as to the activity of the pathway.

According to an article in the American Journal of medicine and critical care by Marcus Bossman, MD at Boston University school of medicine, the complement system is suspected to have a critical role during the development of COVID-19. The article mentions,
"Complement activation may occur early during SARS-CoV-2 infection by the direct interaction of viral proteins with mannosebinding lectin and ficolin pathway. The viral nucleocapsid protein binds to a mannose-binding protein-associated serine protease 2 (MASP-2), a key protease of the MBL pathway leading to overactivation of the complement system and worsening of lung injury. Additionally, the spike protein (S protein) of SARS-CoV-2 may directly bind to ficolins". The production of SARS-CoV-2 specific $\operatorname{IgM}$ and $\operatorname{IgG}$ during approximately the second week of the disease may further activate the complement system via the classical pathway whereas IgA can trigger the MBL pathway per Dr. Marcus Bossman's article [4].

A study on 5 COVID-19 patients with severe symptoms by Cynthia Magro and colleagues [5] further supports the important role complement activation plays in COVID-19 infection [6]. According to this study, skin and lung tissues on autopsy from 5 patients with severe COVID-19 infection characterized by respiratory failure and pruritic skin rash were examined microscopically and with immunohistology. Pulmonary findings were consistent with significant deposits of terminal complement C5b-9, C4d, and MBL associated serum protease in the microvasculature consistent with sustained systemic activation of complement pathways [7]. The pruritic skin lesions also showed a post-inflammatory thrombogenic vasculopathy with deposition of $\mathrm{C} 5 \mathrm{~b}-9$ and $\mathrm{C} 4 \mathrm{~d}$, according to the study

Moreover, in a study using virus-infected C3 -/- mice, by Gralinski and colleagues, complement activation was assessed in SARS-CoV infection with $\mathrm{C} 3$ activation products in the lung as early as one day after SARS-CoV infection. Lung injury and weight loss were significantly reduced in the absence of $\mathrm{C} 3$, despite the unchanged viral load. Substantially fewer neutrophils and inflammatory 
monocytes as well as lower cytokines and chemokine levels were detected in C3 deficient mice. Additionally, the study showed that factor B and C4 deficient mice had less weight loss than wild-type

\section{Case Presentation}

The patient is a 20-year-old Hispanic male without significant past medical history, who tested positive for COVID-19 two weeks before presenting to the emergency department, presented with complaints of worsening shortness of breath, persistent fever, severe coughing spells, as well as multiple near syncopal episodes. He had been self isolating since his positive COVID-19 testing. Vital signs on admission were significant for hypoxia with oxygen saturation of $91 \%$ on room air, tachycardia with a heart rate of 145 , and tachypnea with a respiration rate of 32 and blood pressure of 110/85.

The physical exam revealed an acutely ill-appearing patient lying in his bed. The pulmonary exam was significant for decreased breath sounds bilateral without wheezing, rhonchi, or rales. Cardiac auscultation showed normal S1 and S2 with no murmurs or gallops There was no jugular vein distention or lower extremity edema. Skin examination was negative for any rash, lesions, or ulceration.

Laboratory studies revealed negative COVID-19 testing by PCR. The patient's SARS-CoV-2 antibody testing was positive with the SARSCoV-2 antibody index of greater than 10.0. Complement testing using total hemolytic complement assay (CH50) was greater than 60(normal range from 31-60). C3 was $158 \mathrm{mg} / \mathrm{dL}$ (90 -180 mg/dL) and C4 level was $23 \mathrm{mg} / \mathrm{dL}$ (10 to $40 \mathrm{mg} / \mathrm{dL}$ ). He was found to have elevated troponin of $0.78 \mathrm{ng} / \mathrm{ml}(0.0-0.05 \mathrm{ng} / \mathrm{ml})$ and Pro-Brain Natriuretic peptide of 2103 pg./ml (0- 450 pg./ml). Electrocardiogram showed sinus tachycardia and borderline ST-segment elevation in the anterior leads. Other significant laboratory findings where leukocytosis of 13.7 with $80 \%$ neutrophils and $10 \%$ lymphocytes.

\section{Results and Discussion}

The patient in our case presentation had a positive SARS-CoV-2 antibody with elevated $\mathrm{CH} 50$ in the setting of saddle pulmonary embolism with right heart strain.CH 50 levels are elevated in cases of classical complement activation which correlates with our patient's presentation 2 weeks after initial COVID-19 positive testing. It also is known that complement activation is a prothrombotic state. This shows complement activation due to $\operatorname{IgG} / \operatorname{IgM}$ antibody-viral antigen complex leading to classical complement activation is likely one of the pathophysiologies of COVID-19 infection leading to thrombosis and pulmonary embolism, given patient's positive SARS-CoV-2 antibody at the time of pulmonary embolism. Further studies need to be conducted with more comprehensive complement levels to determine the significance of complement activation immediately after patients are tested positive as well as at a later time during the course of infection(example at 48 hours from admission) as complement testing in our case was performed only two weeks after mice suggesting that complement activation enhances pulmonary pathology and SARS-CoV infection associated with systemic illness.

The patient had elevated inflammatory markers with an erythrocyte sedimentation rate of 44 and a C-reactive protein of $9.0 \mathrm{mg} / \mathrm{dl}(<0.3 \mathrm{mg} / \mathrm{dl})$. Ferritin was elevated at $585 \mathrm{ng} / \mathrm{ml}(26-388$ $\mathrm{ng} / \mathrm{ml})$. Lactate dehydrogenase is $428 \mathrm{U} / \mathrm{L}(87-241 \mathrm{U} / \mathrm{L})$. D-dimer was significantly elevated at greater than $35.2 \mathrm{mg} / \mathrm{L}(0.0-0.49 \mathrm{mg} / \mathrm{L}$ FEU).

A computed tomography angiogram (CTA) thorax revealed extensive bilateral pulmonary emboli including saddle emboli at the bifurcation of the pulmonary trunk extending into both right and left pulmonary arteries as well as bilateral lung infiltrates. Echocardiography showed abnormal septal motion with septal flattening in diastole consistent with right ventricular volume overload.

The patient was started on a heparin drip and bilateral pulmonary artery infusion catheters were placed by interventional radiology for catheter-directed thrombolysis. The patient was then monitored in the intensive care unit. The day following the initiation of catheterdirected thrombolysis, pulmonary angiogram showed minimal residual nonocclusive thrombus in the distal right main pulmonary artery and so thrombolysis was terminated at that time. The patient showed significant clinical improvement after thrombolysis. The patient's anticoagulation was subsequently converted to full dose enoxaparin to bridge to warfarin therapy. The patient was initially placed on empiric antibiotics with cefepime and vancomycin were given bilateral pulmonary infiltrates, however, procalcitonin was negative and antibiotics were discontinued shortly after admission.

positive COVID-19 result.

Early complement testing will give as a more comprehensive picture of the importance of alternative and lectin pathways in the mortality and morbidity of COVID-19 patients. This is due to the unique timing of complement activation for the different pathways with the alternative and lectin pathways activated early in the course of infection.

Based on complement deficient mice study by Granliski and colleagues discussed above, normal, or high complement levels are likely to show worse prognosis in SARS-COV-2 infection. Complement activation not only plays a role in thrombosisbut also plays a role in the extent of adaptive immune response and cytokine storm. Our patient had normal C3 and C4 levels, which placed him at a higher risk of SARS-CoV-2 complication, as patients with complement deficiency are likely to have a better prognosis. Further studies with complement testing on a larger sample population may 
provide more evidence on the prognosis of SARS- COV-2 infection based on complement levels.

Histological findings of five severe COVID-19 patients discussed in the introduction section, which showed significant deposition of complements from lung and skin samples, is great evidence of the significance of complement activation in COVID-19 infection and its

\section{Conclusion}

The most important aspect of this case presentation is to give insight into the importance of classical complement activation in COVID-19 patients and especially concerning thrombotic risk leading to pulmonary embolism, myocardial infarction, and stroke.

In summary, the findings of this case report and literature review are as follows.

1. Classical complement activation likely plays a key role in creating a hypercoagulable state in COVID-19 patients leading to pulmonary embolism, myocardial infarction, and stroke. This likely occurs approximately 2 weeks after the initial infection. It is important to testboth SARS-CoV-2 by PCR as well as the SARSCoV-2 antibody as patients can test negative by PCR but their presentation represents a complication of a previous infection.

2. Complement activation likely plays a role in the pathophysiology of SARS-CoV-2 and its mortality and morbidity because of its role in thrombosis and its effect on adaptive immune response including cytokine storm. Further studies with more extensive complement testing and a larger sample size should be conducted

\section{Acknowledgment}

I would like to acknowledge Dr. Robert Smith for giving me advice and encouraging me for my proposal on a Complement study in COVID-19 patients at Sarasota Memorial Hospital. I would also like to thank Dr. Kirk Voelker for his contribution to facilitating

\section{References}

1. Liszewski MK, Atkinson JP (2020) UpToDate: Complement Pathways.

2. Wolfram Ruf W (2019) Links Between Complement Activation and Thrombosis. Blood 134 (Supplement_1): SCI-40.

3. Costabile M (2010) Measuring the $50 \%$ Haemolytic Complement (CH50) Activity of Serum. Journal of Visualized Experiments 37(37).

4. Bossman M (2020) Complement Activation during Critical Illness: Current Findings and an Outlook in the Era of COVID19: American Journal of Respiratory and Critical Care Medicine 202(2). associated mortality and morbidity. If the significance of complement activation is confirmed based on a larger sample size in different COVID-19 severity groups, clinical trials of complement inhibitors such as compstatin may be considered for the treatment of COVID19 patients with severe symptoms.

to determine the significance of complement activation in mortality and morbidity of SARS-Cov-2 infection especially in cases of severe infection.

3. Complement testing can be used as an early surrogate marker to determine the severity of SARS-CoV-2 infection since the complement system is part of innate immune response and activationis expected (for the alternative and MBL pathways) early in the courseof infection.

4. With further studies, complement inhibitors may be considered for the treatment of severe SARS-CoV-2 infection, including in thrombotic states (pulmonary embolism, stroke), acute lung injury, and multiorgan failure. Complement inhibitors may prevent thrombosis.

5. Although patients are seropositive, this does not mean they are free of SARS-CoV-2 complications, and necessary precautions to preventthrombosis should be taken. For example, taking aspirin or anticoagulation in high-risk patients.

complement testing on the patient. Equally important, I would like to acknowledge Dr. Sary Beidas for his time in reviewing the article and giving me information on literature about my topic.

Disclosure of Conflicts of Interest: There are no conflicts of interest.

5. Magro C, Mulvey JJ, Berlin D, Nuovo G, Salvatore S, et al. (2020) Complement associated microvascular injury and thrombosis in the pathogenesis of severe COVID-19 infection: Areport of five cases. Transl Res 220: 1-13.

6. Chapin J,Terry HS, Kleinert D, Laurence J (2016) The Role of Complement Activation in Thrombosis and Hemolytic Anemias. Transfusion and Apheresis Science 54(2): 191-198.

7. Jason MS, Julia RC, Ernest EM, Matt B, Navin V, et al. (2019) Alternative Complement Pathway Activation Provokes a Hypercoagulable State with Diminished Fibrinolysis. Shock 53(5): 560-565. 